\title{
On PERIODICAL OSCILLATIONS of LURIE SYSTEMS with DISCONTINUOUS NONLINEARITY
}

\section{Efimov D.V.}

\author{
Systems and Control, Université de Liège, Bat. 28, B-4000 Liege Sart-Tilman, BELGIUM \\ Institute for Problem of Mechanical Engineering, 61, Bolshoy av., V.O. , St-Petersburg, 199178 Russia
}

\begin{abstract}
Sufficient conditions of global attracting limit cycle existence for Lurie system with sign nonlinearity are presented. It is assumed that the linear part of the system is output stabilizable, the nonlinearity has linear negative term plus positive one proportional to the output sign. Conditions of oscillatority in the sense of Yakubovich for this class of systems are also reestablished. Copyright (C) 2008 IFAC
\end{abstract}

Keywords: Switching stability and control, Analytic design, Output feedback control.

\section{INTRODUCTION}

The problem of stability of Lurie type systems (i.e. the systems which are composed by linear subsystem closed by nonlinear output feedback) has seen a lot of attention during the last more than 60 years. This type of systems firstly appeared in paper of Bulgakov (1942), where the problem of absence of self-sustained oscillations was considered, and the system received its name after the famous work of Lurie and Postnikov (1944).

The conditions of asymptotic stability of the origin was firstly proposed in (Yakubovich, 1962; Kalman, 1963; Popov, 1962) and (Sandberg, 1964; Zames, 1966), and after that it had many extensions (Aizerman and Gantmacher, 1964; Narendra and Taylor, 1973). More complex problem of the solutions boundedness was widely addressed starting from (Yakubovich, 1965) (see also Răsvan (2007) in special issue (2007) for the latest achievements in the field of Lurie systems stability), in paper (Arcak and Teel, 2002) the inputto-state stability conditions were obtained for Lurie systems with external inputs.

In papers (Yakubovich, 1964; 1966) problem of forced (periodical) oscillations existence was posed and solved by Prof. V.A. Yakubovich. In a few years later he introduced (1973) a new definition of self-sustained oscillations (or auto-oscillation) and proposed conditions of this type of oscillations existence for Lurie systems (Yakubovich, 1975; Yakubovich and Tomberg, 1989) (in work (Yakubovich, 1975) conditions of oscillatority for systems with discontinuous at the origin nonlinearity were presented). This type of oscillatority later received the name of Prof. Yakubovich (Leonov, et al., 1995). Recently those conditions of the oscillatority were extended to generic nonlinear systems decomposed in nonlinear dynamical subsystem closed by static nonlinear output feedback (Efimov and Fradkov, 2004; 2007).

The oscillatority in the sense of Yakubovich is rather general conception which covers periodical, non periodical as well as chaotic oscillations. This is why very frequently the establishing of this property is not enough, since it does not provide information about kind of oscillations (estimates on oscillation amplitude can be obtained and in some cases frequency spectrum lower estimate can be derived), and further investigations on periodical oscillations existence were carried out. In works (Yakubovich 1964; 1966; Bliman and Krasnosel'skii, 1998) series of conditions for forced periodical oscillations existence were presented (in papers (Bliman and Krasnosel'skii, 1998) time-dependent periodical nonlinearity was considered). In works (Krasnosel'skii and Rachinskii, 2002; Stan and Sepulchre, 2005; 2007) autonomous Lurie system was considered and existence of limit cycle was proven applying bifurcation theory, that results to local conditions of limit cycle existence. In papers (Aguilar, et al., 2007; Bliman, et al., 2000; Loh and Vasnani, 1994) authors applied describing functions like approaches for limit cycle existence substantiation (in paper (Bliman, et $a l ., 2000)$ continuous nonlinearity was considered only), that provides easily verified practical frequency domain conditions of oscillations existence. However, describing function approach (Atherton, 1975) does not provide an exact 
answer on question about periodical oscillations existence since the approach starts with assumption that solutions of the system are harmonic functions of time, that is not a correct assumption for the nonlinear Lurie system (the possibility of asymmetric oscillations existence for Lurie system with sign nonlinearity was analyzed in (Di Bernardo, et al., 2001), see also (Kuznetsov, 1995)). Existence of limit cycles for Lurie system with sign nonlinearity was considered in paper (Di Bernardo, et al., 2001) applying Poincaré map approach (that is also a local technique). In paper (Efimov and Fradkov, 2007) frequency of oscillations were derived for the second order system (existence of limit cycles for second order systems follows from PoincaréBendixson theorem and its extensions (Mallet-Paret and Sell, 1996)).

Much more attention in the literature was devoted to Lurie systems with stable linear part closed by nonlinearity proportional to negative sign of the output, that is a typical situation in PID control systems with relays (Johansson, et al., 2002; Tsypkin, 1984). In paper (Megretski, 1996) conditions for global limit cycle existence and stability were firstly formulated for this type of systems, in this paper it was assumed that linear part of the system has unit step response close in some sense to non minimum phase second order linear stable system. An approach to establish the existence of globally stable limit cycle for piecewise linear systems is presented in work (Goncalves, et al., 2003).

Thus, despite availability of wide range oscillatority theory (Andronov, et al., 1965; Bogolyubov and Mitropolsky, 1962; Nemytskii and Stepanov, 1989), for the best of our knowledge there is no global results on global limit cycle existence (globally attracting periodical invariant solution) for Lurie systems with locally positive feedback. In this paper we are going to develop results from (Efimov and Fradkov, 2007) for Lurie systems with positive sign nonlinearity feedback with order bigger than 2. Auxiliary definitions and statements are presented in section 2. Section 3 contains conditions of the system oscillatority in the sense of Yakubovich (considering Lurie system with particular type of nonlinearity we propose more constructive conditions than obtained in (Yakubovich, 1975), additionally upper and lower estimates on oscillations amplitude are derived). Periodicity of the system solutions is investigated in section 4. Example is presented in section 5.

\section{PRELIMINARIES}

Consider SISO linear time-invariant finite dimensional system:

$$
\dot{\mathbf{x}}=\mathbf{A} \mathbf{x}+\mathbf{b} u, y=\mathbf{c}^{T} \mathbf{x},
$$

where $\mathbf{x} \in R^{n}$ is state, $y \in R$ is output, $u \in R$ is input, signal $u: R_{+} \rightarrow R$ is piecewise continuous function of time $t \in R_{+}, R_{+}=\{t \in R: t \geq 0\}, R$ is Euclidean space; $\mathbf{A}$ is $n \times n$ matrix with real elements, $\mathbf{b}$ and $\mathbf{c}$ are vectors in $R^{n}$. A s s u mption 1. Let system (1) be stabilizable by static output $y$ feedback and have relative degree 1 , i.e. there exist symmetric positive definite matrix $\mathbf{P}$ with dimension $n \times n$ and $k>0$ such, that

$$
\mathbf{G}^{T} \mathbf{P}+\mathbf{P} \mathbf{G}+\alpha \mathbf{I}_{n} \leq 0, \mathbf{G}=\mathbf{A}-k \mathbf{b} \mathbf{c}^{T}, \mathbf{c}^{T} \mathbf{b}>0,
$$

where $\alpha$ is some positive constant and $\mathbf{I}_{n}$ is identity matrix with dimension $n \times n$.

Note that by the standard results (see (Astolfi and Colaneri, 2000), for example) in this case matrices pairs (A, b) is controllable and $\left(\mathbf{A}, \mathbf{c}^{T}\right)$ is observable. Requirement on relative degree is introduced for simplicity of consideration and the case with high relative degree can be treated in the same way. Under this assumption for system (1) with input $u=-k y$ there exists Lyapunov function $V(\mathbf{x})=\mathbf{x}^{T} \mathbf{P} \mathbf{x}$ with time derivative $\dot{V} \leq-\alpha|\mathbf{x}|^{2}$. Consider feedback input

$$
\begin{aligned}
u & =-k y+\operatorname{sign}(y), \\
\operatorname{sign}(y) & =\left\{\begin{array}{c}
y /|y| \text { if }|y| \neq 0 ; \\
0 \text { otherwise, }
\end{array}\right.
\end{aligned}
$$

where we have linear negative output feedback item and positive discontinuous feedback one formed by sign function. Our interest in such form of feedback follows from the fact, that dynamics of many biological, chemical or physical oscillating systems can be expressed in terms of Lurie system (1), (2) (Efimov and Fradkov, 2004; Stan and Sepulchre, 2005), that is passive system (matrix A is stable (Zames, 1966)) closed by feedback, which has globally stabilizing feedback term plus locally destabilizing term.

Let for any initial value $\mathbf{x}_{0} \in R^{n}$ the solution of system (1), (2) be denoted as $\mathbf{x}\left(t, \mathbf{x}_{0}\right)$ if it satisfies (1), (2) for almost all $0 \leq t \leq T<+\infty$, by standard arguments it is absolutely continuous and defined at least locally (Filippov, 1988) $\left(y\left(t, \mathbf{x}_{0}\right)=\mathbf{c}^{T} \mathbf{x}\left(t, \mathbf{x}_{0}\right)\right.$, we also will write $\mathbf{x}(t)$ and $y(t)$ if origin of initial condition is clear from the context). If for all initial conditions $\mathbf{x}_{0} \in R^{n}$ the corresponding solutions $\mathbf{x}\left(t, \mathbf{x}_{0}\right)$ are defined for all $t \geq 0$, then the system is called forward complete. Now we are in position to define the property of oscillatority in the sense of Yakubovich for system (1), (2).

Definition 1 (Yakubovich, 1973). Solution $\mathbf{x}\left(t, \mathbf{x}_{0}\right)$ with $\mathbf{x}_{0} \in R^{n}$ of system (1),(2) is called $\left[\pi^{-}, \pi^{+}\right]$oscillation with respect to output $\psi=\eta(\mathbf{x})$ (where $\eta: R^{n} \rightarrow R$ is a continuous monotonous with respect to all arguments function) if the solution is defined for all $t \geq 0$ and for $-\infty<\pi^{-}<\pi^{+}<+\infty$

$$
\varliminf_{t \rightarrow+\infty} \psi\left(t, \mathbf{x}_{0}\right)=\pi^{-} ; \varlimsup_{t \rightarrow+\infty} \psi\left(t, \mathbf{x}_{0}\right)=\pi^{+} .
$$

Solution $\mathbf{x}\left(t, \mathbf{x}_{0}\right)$ with $\mathbf{x}_{0} \in R^{n}$ of system (1),(2) is called oscillating, if there exist some output $\psi$ and constants $\pi^{-}$, 
$\pi^{+}$such, that $\mathbf{x}\left(t, \mathbf{x}_{0}\right)$ is $\left[\pi^{-}, \pi^{+}\right]$-oscillation with respect to the output $\psi$. Forward complete system (1), (2) is called oscillatory, if for almost all $\mathbf{x}_{0} \in R^{n}$ the solutions of the system $\mathbf{x}\left(t, \mathbf{x}_{0}\right)$ are oscillating. Oscillatory system (1) is called uniformly oscillatory, if for almost all $\mathbf{x}_{0} \in R^{n}$ for corresponding solutions $\mathbf{x}\left(t, \mathbf{x}_{0}\right)$ there exist common output $\psi$ and constants $\pi^{-}, \pi^{+}$not depending on initial conditions.

Function $\eta: R^{n} \rightarrow R$ is monotonous if it is monotonous for each particular scalar argument for all fixed values of other arguments. Oscillatority property is introduced for almost all initial conditions since system (1), (2) has some equilibrium points, stating from which the system solutions are not oscillating.

Assumption 2. Let

$$
\operatorname{sign}\left[\mathbf{c}^{T}\left(\mathbf{A}-k \mathbf{b c}^{T}\right)^{-1} \mathbf{b}\right] \geq 0 .
$$

That is a technical condition which will be used in the next section to prove uniqueness of the equilibrium in the origin for system (1), (2).

\section{YAKUBOVICH's OSCILLATORITY}

We are interesting in uniform oscillatority conditions for system (1), (2). To do so we should prove uniqueness of equilibrium in the origin (it is obvious that it is an equilibrium for the system (1), (2)), global boundedness of the system solutions and local instability of the equilibrium.

Prop os ition 1. Let assumptions 1, 2 hold, then system (1), (2) has the single equilibrium $\mathbf{x}=0$.

Proofs are excluded due to space limitations.

Proposition 2. Let assumption 1 hold, then for all initial conditions $\mathbf{x}_{0} \in R^{n}$ the solutions of system (1), (2) $\mathbf{x}\left(t, \mathbf{x}_{0}\right)$ obey the estimates:

$$
\begin{aligned}
& \left|\mathbf{x}\left(t, \mathbf{x}_{0}\right)\right| \leq \sqrt{2 \frac{\lambda_{\max }(\mathbf{P})}{\lambda_{\min }(\mathbf{P})}}\left[\left|\mathbf{x}_{0}\right| \times\right. \\
& \left.\times e^{-0.25 \alpha \lambda_{\max }(\mathbf{P})^{-1} t}+\sqrt{2} \alpha^{-1}|\mathbf{P} \mathbf{b}|\right], t \geq 0, \\
& \varlimsup_{t \rightarrow+\infty}\left|\mathbf{x}\left(t, \mathbf{x}_{0}\right)\right| \leq R, R=2 \sqrt{\frac{\lambda_{\max }(\mathbf{P})}{\lambda_{\min }(\mathbf{P})}} \alpha^{-1}|\mathbf{P} \mathbf{b}|,
\end{aligned}
$$

where $\lambda_{\min }(\mathbf{P})$ and $\lambda_{\max }(\mathbf{P})$ are minimal and maximal eigen-values of matrix $\mathbf{P}$.

In fact this proposition is a corollary of results from (Arcak and Teel, 2002), where more generic result on Lurie systems solutions boundedness was proven. Proposition 2 is presented since we need further some estimates derived there.

Proposition 3 . Let assumption 2 hold, then the origin $\mathbf{x}=0$ is unstable equilibrium for system (1), (2), moreover for all initial condition $\mathbf{x}_{0} \in R^{n} /\{0\}$ it holds that

$$
\begin{gathered}
\varliminf_{t \rightarrow+\infty}\left|\mathbf{x}\left(t, \mathbf{x}_{0}\right)\right| \geq r, \\
r=\frac{k^{-1}|\mathbf{c}|^{-1}}{1+k^{-1}|\mathbf{c}|^{-1}\left(\mathbf{c}^{T} \mathbf{b}\right)^{-1}\left|\mathbf{c}^{T} \mathbf{A}\right|} .
\end{gathered}
$$

Summarizing the discussion presented so far note that under assumptions 1 and 2 all trajectories outside the origin converge to set

$$
\begin{gathered}
\Omega=\left\{\mathbf{x} \in R^{n}: r \leq|\mathbf{x}| \leq R\right\}, \\
r=\frac{k^{-1}|\mathbf{c}|^{-1}}{1+k^{-1}|\mathbf{c}|^{-1}\left(\mathbf{c}^{T} \mathbf{b}\right)^{-1}\left|\mathbf{c}^{T} \mathbf{A}\right|}, \\
R=2 \sqrt{\frac{\lambda_{\text {max }}(\mathbf{P})}{\lambda_{\text {min }}(\mathbf{P})}} \alpha^{-1}|\mathbf{P} \mathbf{b}| .
\end{gathered}
$$

Now we are ready to describe new oscillatority conditions for Lurie system (1), (2).

The o r e m 1. Let assumption 1, 2 hold and $\mathbf{c}^{T} \mathbf{G}^{-1} \mathbf{b} \neq 0$ if all eigen-values of matrix $\mathbf{G}$ are real. Then system (1), (2) is an oscillating one and for almost all initial conditions $\mathbf{x}_{0} \in R^{n} /\{0\}$ the solutions $\mathbf{x}\left(t, \mathbf{x}_{0}\right)$ are $\left[\pi^{-}, \pi^{+}\right]-$ oscillations with respect to common output $y$, where

$$
-|\mathbf{c}| R \leq \pi^{-}<\pi^{+} \leq|\mathbf{c}| R .
$$

Re mark 1 . New condition $\mathbf{c}^{T} \mathbf{G}^{-1} \mathbf{b} \neq 0$ (if all eigenvalues of matrix $\mathbf{G}$ are real) excludes from consideration the confluent case, when formally system has an attracting invariant compact subset into $\Omega$ (without equilibriums of system (1), (2)), but equilibriums of systems

$$
\dot{\mathbf{x}}=\mathbf{G} \mathbf{x}+s \mathbf{b}, s= \pm 1,
$$

lie exactly on switching surface where $\mathbf{c}^{T} \mathbf{x}=0$ inside the set $\Omega$. If all eigen-values are real, then in general case there exist subsets of initial conditions such, that trajectories converge to equilibriums $\mathbf{x}^{0}=-s \mathbf{G}^{-1} \mathbf{b}, s= \pm 1$ of systems (3) without any intersections of surface $\mathbf{c}^{T} \mathbf{x}=0$. Asymptotically they reach for equilibriums $\mathbf{x}^{0}$ where switches occur. System trajectory will have no exact limits in this case and it is oscillating with infinite time "period".

\section{LIMIT CYCLE EXISTENCE}

In general case oscillatority in the sense of Yakubovich implies that system is oscillating possibly with many stable or unstable closed trajectories. Further we are interesting in the case when the system has the single globally stable limit cycle which is obviously belongs to set $\Omega$ (limit cycle is the set of values of an isolated periodic trajectory, which is not an equilibrium). Limit cycle is globally stable if almost all trajectories of the system asymptotically converge to it. Let us start with simple statement on globally stable limit cycle existence for second order system.

Proposition 4. Let $n=2$, assumptions 1,2 hold, $\mathbf{c}^{T} \mathbf{G}^{-1} \mathbf{b}=0$ and matrix $\mathbf{G}$ have two complex conjugate 
eigen-values $\lambda_{1,2}=-\alpha \pm \omega i$. Then under conditions of theorem 1 the system has globally stable limit cycle and

1. The limit cycle has period $T=2 \pi \omega^{-1}$.

2. The limit cycle has finite time of convergence.

The proof is straightforward and it is based on direct computations, it is excluded due to space requirements. Under conditions of proposition 2 the system has symmetric globally stable limit cycle. This limit cycle is robust with respect to additive asymptotically vanishing disturbances as it is stated in the following theorem.

The orem 2. Let assumption 1, 2 hold and there exist nonsingular matrix $\mathbf{R} \in R^{n \times n}$ such, that

$$
\begin{gathered}
\tilde{\mathbf{G}}=\mathbf{R} \mathbf{G ~ R}^{-1}=\left[\begin{array}{ccc}
\tilde{\mathbf{G}}_{1,1} & \mathbf{0}_{n_{1} \times 2} & \mathbf{0}_{n_{1} \times n_{3}} \\
\tilde{\mathbf{G}}_{2,1} & \tilde{\mathbf{G}}_{2,2} & \mathbf{0}_{2 \times n_{3}} \\
\tilde{\mathbf{G}}_{3,1} & \tilde{\mathbf{G}}_{3,2} & \tilde{\mathbf{G}}_{3,3}
\end{array}\right], \\
\tilde{\mathbf{b}}=\mathbf{R} \mathbf{b}=\left[\begin{array}{c}
\mathbf{0}_{n_{1} \times 1} \\
\tilde{\mathbf{b}}_{2} \\
\tilde{\mathbf{b}}_{3}
\end{array}\right], \tilde{\mathbf{c}}=\left(\mathbf{R}^{-1}\right)^{T} \mathbf{c}=\left[\begin{array}{c}
\tilde{\mathbf{c}}_{1} \\
\tilde{\mathbf{c}}_{2} \\
\mathbf{0}_{n_{3} \times 1}
\end{array}\right], \\
\tilde{\mathbf{c}}_{2}^{T} \tilde{\mathbf{G}}_{2,2}^{-1} \tilde{\mathbf{b}}_{2}=0,
\end{gathered}
$$

where $\mathbf{0}_{n_{1} \times n_{3}}$ denotes the matrix of zeros with dimension $n_{1} \times n_{3}, \quad \tilde{\mathbf{G}}_{2,2} \in R^{2 \times 2}, \quad \tilde{\mathbf{b}}_{2} \in R^{2}, \quad \tilde{\mathbf{c}}_{2} \in R^{2}, n_{1}+2+n_{3}=n$ (all other matrices have corresponding dimensions). If matrix $\tilde{\mathbf{G}}_{2,2}$ has two complex conjugate eigen-values, then the system (1), (2) has globally stable limit cycle.

Conditions of theorem 2 describes a scenarios of globally stable limit cycle arising. In this case Lurie system (1), (2) can be recomposed on three subsystems as it is in particular shown in Fig. 1. System 2 (which corresponds to dynamics of variable $\mathbf{z}_{2}$ ) plays a role of "clock" for the system producing globally stable limit cycle and defining period of oscillations. Discovering other ways of limit cycle arising for system 2 one can characterize different situations of globally stable limit cycle existence for Lurie systems.

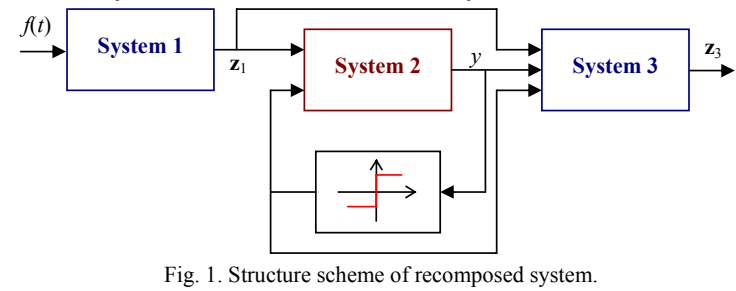

R e mark 2. It is clear that the result of theorem will be preserved for any bounded and asymptotically converging to zero external disturbance $f: R_{+} \rightarrow R$ (it is supposed that $f$ is Lebesgue measurable and essentially bounded function of time $t \geq 0)$. Moreover solutions of the system will be bounded for any such bounded $f$. Applying excitation indices approach from (Efimov and Fradkov, 2004) one can investigate robustness of oscillatority property of the system against to $f$.

R e mark 3 . System 1 in Fig. 1 can be replaced with any nonlinear globally asymptotically stable system with saving previously obtained conclusions about the system properties. System 3 also can be replaced with Lurie system (in paper (Yakubovich, 1966) the conditions were derived, which ensure existence of unique periodical solution for Lurie system with periodical external input) or with nonlinear convergent system, which also has unique periodical response on periodic input (see (Pavlov, et al., 2005) for convergent systems approach).

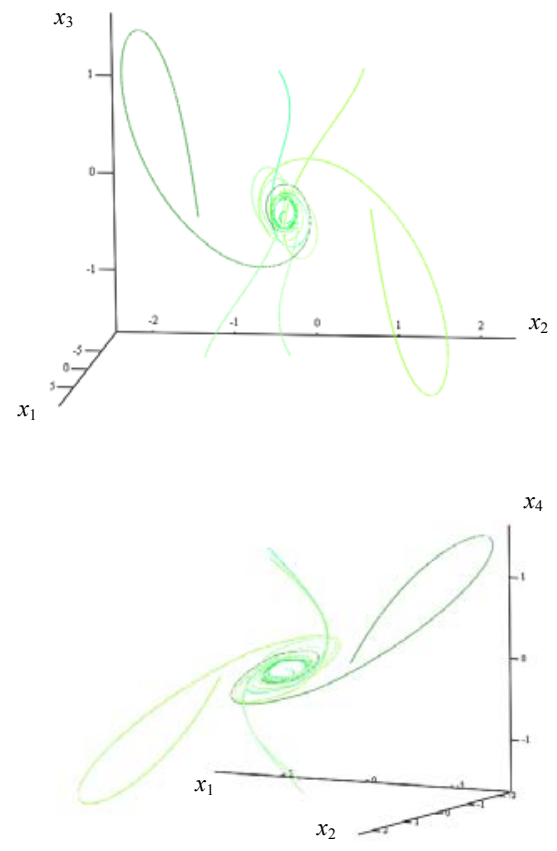

Fig. 2. Results of simulations.

\section{SIMULATIONS}

Consider system (1), (2) with the following values of all parameters for $n=4$ :

$$
\begin{gathered}
\mathbf{A}=\left[\begin{array}{cccc}
-3.333 & 2.333 & -7.778 & 3.667 \\
-0.333 & -0.667 & 0.222 & 0.667 \\
1 & -1 & -1.333 & -1 \\
-2.667 & 2.667 & -7.889 & 2.333
\end{array}\right], \\
\mathbf{b}=\left[\begin{array}{c}
-1 \\
0 \\
0 \\
-1
\end{array}\right], \mathbf{c}=\left[\begin{array}{c}
0 \\
-1 \\
2 \\
-1
\end{array}\right], k=1
\end{gathered}
$$

Matrix A is stable and it has eigen-values [1.414i $-1.414 i-2-1]$ (system (1) is passive), $\mathbf{c}^{T} \mathbf{b}=1$, $\alpha=1$ and

$$
\mathbf{G}=\left[\begin{array}{cccc}
-3.333 & 1.333 & -5.778 & 2.667 \\
-0.333 & -0.667 & 0.222 & 0.667 \\
1 & -1 & -1.333 & -1 \\
-2.667 & 1.667 & -5.889 & 1.333
\end{array}\right],
$$




$$
\mathbf{P}=\left[\begin{array}{cccc}
11.48 & -11.389 & 4.440 & -11.054 \\
-11.389 & 12.024 & -4.173 & 11.157 \\
4.440 & -4.173 & 7.049 & -6.016 \\
-11.054 & 11.157 & -6.016 & 11.607
\end{array}\right]
$$

Therefore, all conditions of assumption 1 are satisfied. Since $\operatorname{sign}\left[\mathbf{c}^{T}\left(\mathbf{A}-k \mathbf{b c}^{T}\right)^{-1} \mathbf{b}\right] \geq 0$, then assumption 2 is also valid. Matrix G has eigen-values $[-0.5+1.323 i-0.5-1.323 i-2-1]$ and all conditions of theorem 1 are satisfied, thus system is oscillating. To apply theorem 2 let us choose

$$
\mathbf{R}=\left[\begin{array}{cccc}
0.556 & -0.333 & -0.556 & 0.444 \\
-0.444 & -0.333 & -0.556 & -0.556 \\
0.667 & 0 & 0.333 & 0.333 \\
1.778 & 0.333 & 0.222 & 1.222
\end{array}\right],
$$

then $n_{1}=n_{3}=1$ and

$$
\tilde{\mathbf{G}}=\left[\begin{array}{cccc}
-1 & 0 & 0 & 0 \\
2 & 0 & 1 & 0 \\
-1 & -2 & -1 & 0 \\
-2 & 1 & -1 & -2
\end{array}\right], \tilde{\mathbf{b}}=\left[\begin{array}{c}
0 \\
0 \\
1 \\
-1
\end{array}\right], \tilde{\mathbf{c}}=\left[\begin{array}{l}
0 \\
0 \\
1 \\
0
\end{array}\right]
$$

and matrix $\tilde{\mathbf{G}}_{2,2}$ has eigen-values $[-0.5+1.323 i-0.5-1.323 i]$. Therefore, according to theorem 2 the system has globally stable limit cycle. The results of the system simulation are shown in Fig. 2, where projection of the system trajectories on two hyper planes are presented. As it can be seen from Fig. 2 the system has limit cycle that approves the results of the paper.

\section{CONCLUSION}

Conditions of oscillatority in the sense of Yakubovich are revisited for Lurie system with sign nonlinearities, additionally a scenarios of globally stable limit cycle arising in Lurie systems is described and characterized. Computer simulations approves analytical results of the paper.

\section{ACKNOWLEDGEMENT}

Authors would like to thank Prof. E. Panteley for fruitful discussion and comments.

\section{REFERENCES}

Aizerman, M. A., \& Gantmacher, F. R. (1964). Absolute stability of regulator systems. San Francisco: HoldenDay. (Translated from the Russian original, Moscow: Akademiya Nauk SSSR, 1963).

Aguilar L.T., Boiko I., Fridman L., Iriarte R. (2007). Periodic Motion of Underactuated Mechanical Systems Selfgenerated by Variable Structure Controllers: Design and
Experiments. Proc. European Control Conference 2007, Kos, Greece, pp. 3796-3801.

Andronov A. A., Khaikin S. E., Vitt A. A. Theory of Oscillators. Pergamon Press, Oxford, 1965.

Arcak M., Teel A. (2002). Input-to-state stability for a class of Lurie systems. Automatica, 38, pp. 1945 - 1949.

Astolfi A., Colaneri P. (2000). Static output feedback stabilization of linear and nonlinear systems. Proc. 39th IEEE Conference on Decision and Control 2000, pp. 2920-2925.

Atherton D.P. (1975). Nonlinear control engineeringDescribing Function Analysis and Design. Workingham, U.K.: Van Nostrand.

Bliman P.A., Krasnosel'skii A.M. (1998). Popov-like frequency criterion for existence of forced periodic oscillations. Proc. 37th IEEE Conference on Decision and Control, Tampa, Florida, TM 12-7, pp. 2467-2472.

Bliman P.-A., Krasnosel'skii A.M., Rachinskii D.I. (2000). Sector estimates of nonlinearities and self-oscillation existence in control systems. Report 00-002, National University of Ireland, University College, Cork, March 2000, pp. 1-20.

Bogolyubov N. N., Mitropolsky Y.A. (1962). Asymptotic Methods in the Theory of Nonlinear Oscillations. Gordon and Breach, New York, 1962.

Bulgakov B.V. (1942). Self-sustained oscillations of control systems. Doklady Akademii Nauk SSSR, 37(9), pp. 283287 (in Russian).

Di Bernardo M., K.H. Johansson, Vasca F. (2001). Selfoscillations and sliding in relay feedback systems: Symmetry and bifurcations. Int. J. of Bifurcation and Chaos, 11, pp. 1121-1140.

Efimov D., Fradkov A.L. (2004). Excitation of oscillations in nonlinear systems under static feedback. Proc. 43 IEEE Conference on Decision and Control, Dec. 15-18, 2004, pp. $2521-2526$.

Efimov D.V., Fradkov A.L. (2007). Oscillatority Conditions for Nonlinear Systems with Delays. Journal of Applied Mathematics, 2007, Article ID 72561.

Efimov D.V., Fradkov A.L. (2007). Hybrid adaptive resonance control using speed gradient approach for vibration machines. Proc. American Control Conference 2007, New-York, USA.

Filippov A.F. (1988). Differential Equations with Discontinuous Righthand Sides. Kluwer Academic Publishers.

Goncalves J., Megretski A., Dahleh M. (2003). Global analysis of piecewise linear systems using impact maps and quadratic surface Lyapunov functions. IEEE Transactions on Automatic Control, 48, pp. 2089-2106.

Johansson K. H., Rantzer A., Åström K. J. (1999). Fast switches in relay feedback systems. Automatica, 35(4), pp. 539-552.

Johansson K. H., Barabanov A.E., Åström K. J. (2002). Limit cycles with chattering in relay feedback systems. IEEE Transactions on Automatic Control, 47(9), pp. 14141423.

Kalman R.E. (1963). Liapunov functions for the problem of Lur'e in automatic control. Proceedings of the National 
Academy of Sciences of the United States of America, 49(2), pp. 201-205.

Krasnosel'skii A.M., Mennicken R., Rachinskii D.I. (1999). Small periodic solutions generated by sublinear terms. Report 99-009, National University of Ireland, University College, Cork, October 1999, pp. 1-30.

Krasnosel'skii A.M., Rachinskii D.I. (2002). On existence of cycles in autonomous systems. Doklady Mathematics, 384(2), pp. 161-166.

Kuznetsov Y. (1995). Elements of Applied Bifurcation Theory. Springer-Verlag.

Leonov G.A., Burkin I.M., Shepelyavyi A.I. (1995). Frequency Methods in Oscillation Theory. Kluwer, Dordrecht. (in Russian: 1992).

Lurie A.I., Postnikov V.N. (1944). On the theory of stability for control systems. Prikladnaya Matematika $i$ Mekhanika (PMM), 8(3), pp. 246-248 (in Russian).

Loh A.P., Vasnani V.U. (1994). Describing function matrix for multivariable systems and its use in multiloop PI design. J. Process Control, 4, pp. 115-120.

Mallet-Paret J. and Sell G.R. (1996). The PoincaréBendixson Theorem for monotone cyclic feedback systems with delay. J. Differential Equations, 125, pp. $441-489$.

Megretski A. (1996). Global stability of oscillations induced by a relay feedback. Proc. 13th IFAC World Congress 1996, Vol. E, San Francisco, CA, pp. 49-54.

Narendra, K. S., Taylor, J. (1973). Frequency domain methods in absolute stability. New York: Academic Press.

Nemytskii V.V., Stepanov V.V. (1989). Qualitative Theory of Differential Equations. Dover, New York.

Popov V.M. (1962). Absolute stability of nonlinear control systems of automatic control. Automation and Remote Control, 22, pp. 857-875.

Pavlov A., N. van de Wouw and Nijmeijer H. (2005). Uniform Output Regulation of Nonlinear Systems: A convergent Dynamics Approach. Boston: Birkhauser.

Răsvan V. (2007). A new dissipativity criterion - towards Yakubovich oscillations. Int. Journal of Robust and Nonlinear Control, 17(5-6), pp. 483-495.

Sandberg I. W. (1964). A frequency domain condition for the stability of systems containing a single time-varying nonlinear element. The Bell System Technical Journal, 43, pp. 1601-1638.

Special Issue: Frequency-domain and Matrix Inequalities in Systems and Control Theory Dedicated to the 80th Birthday of V. A. Yakubovich. (2007). Int. Journal of Robust and Nonlinear Control, 17(5-6), Issue Edited by A.L. Fradkov, G. Leonov.

Stan G.-B., Sepulchre R. (2005). Feedback Mechanisms for Global Oscillations in Lure Systems. Systems \& Control Letters, 54(8), pp. 809-818.

Stan G.-B., Sepulchre R. (2007). Analysis of interconnected oscillators by dissipativity theory. IEEE Transactions on Automatic Control 2007, 52(2), pp. 256-270.

Tsypkin Ya. Z. (1984). Relay Control Systems. Cambridge University Press, Cambridge, UK.
Yakubovich V.A. (1962). Solution of some matrix inequalities met in control theory. Doklady Akademii Nauk SSSR, 143(6), pp. 1304-1307 (in Russian).

Yakubovich V.A. (1964). LMI in stability theory of nonlinear control systems. Part I: Absolute stability of forced oscillations. Automation and Remote Control, 7, pp. 1-13.

Yakubovich V.A. (1965). Frequency domain criteria for absolute stability and dissipativity of control systems with a differentiable nonlinearity. Doklady Akademii Nauk SSSR, 160(2), pp. 298-301 (in Russian).

Yakubovich V.A. (1966). Periodic and almost periodic limit regimes of control systems with several, generally speaking, discontinuous elements. Doklady Akademii Nauk SSSR, 171(3), pp. 533-536 (in Russian).

Yakubovich V.A. (1973). Frequency oscillations conditions in nonlinear systems with stationary single nonlinearity. Siberian math journal, 14, 2.

Yakubovich V.A. (1975). Oscillations in systems with discontinuous and hysteresis nonlinearities. Automation and Remote Control, 12, pp. 1-10.

Yakubovich V.A., Tomberg E.A. (1989). Conditions for selfinduced oscillations in nonlinear systems, Siberian Math. J., 30, pp. 641-653.

Zames, G. (1966). On the input-output stability of timevarying nonlinear feedback systems-Parts I and II. IEEE Transactions on Automatic Control, 11, pp. 228238 and $465-476$. 